\title{
Chaotic-Search-Based Cultural Algorithm for Solving Unconstrained Optimization Problem
}

\author{
Jianjia $\mathrm{He}^{1,2}$ and Fuyuan $\mathrm{Xu}^{1,2}$ \\ ${ }^{1}$ Business School, University of Shanghai for Science and Technology, Shanghai 200093, China \\ ${ }^{2}$ Center for Supernetworks Research (China), Shanghai 200093, China
}

Correspondence should be addressed to Fuyuan Xu, xufy@usst.edu.cn

Received 16 March 2011; Revised 25 May 2011; Accepted 29 June 2011

Academic Editor: Farouk Yalaoui

Copyright ( $\odot 2011$ J. He and F. Xu. This is an open access article distributed under the Creative Commons Attribution License, which permits unrestricted use, distribution, and reproduction in any medium, provided the original work is properly cited.

\begin{abstract}
For premature convergence and instability of cultural algorithm in solving function optimization problem, based on cultural algorithm and chaos search optimization, a chaos cultural algorithm (CCA) is proposed. The algorithm model consists of a chaosbased population space and a knowledge-storing belief space, uses normative knowledge and situational knowledge for chaos search and chaos perturbation, respectively, effectively avoids premature convergence of cultural algorithm, and overcomes chaos search optimization's sensitivity to initial values and poor efficiency. Test results show that this algorithm is strong in global search and has good performance in searching efficiency, precision, and stability, especially in solving high-dimensional optimization problem.
\end{abstract}

\section{Introduction}

Since the found of the bionics in the 1950s, people began to simulate the evolution mechanism of creatures and proposed such evolutionary computation approaches as the genetic programming and immune algorithm to solve the optimization problem. These algorithm researches are mainly focused on the level of natural choice. Many practical cases show that culture can make population evolve and adapt to the environment at certain speed beyond the evolutionary speed which is merely based on the genetic inheritance [1]. In the human society, culture is regarded as the store carrier of information which is widely delivered among and inside the social groups and can be inherited by all the social members so as to effectively guide them to solve problems. Enlightened from this, Reynolds put forward a brand-new evolutionary computation of cultural algorithm [1].

The cultural algorithm simulates the evolution from both the macroscopic and microscopic levels which stimulate and influence each other mutually. This algorithm provides a kind of explicit mechanism for acquiring, preserving, and integrating the solution knowledge so as to improve the search efficiency. Nowadays, the cultural algorithm, as the hotspot of the algorithm research, has been successfully applied to many fields and achieved better results at certain problems than the traditional evolution algorithm [2-4]. As for the function optimization, the cultural algorithm possesses high solution efficiency, but it has such deficiencies as premature convergence, instability, and so on. The chaotic search optimization is characterized with high global search ability, but it is sensitive to the initial values and poor in searching efficiency. Therefore, this study puts forward the chaos cultural algorithm (CCA) by introducing the chaotic search optimization into the cultural algorithm model. Because of taking advantage of the stochastic and ergodic properties of the chaotic search optimization, this algorithm increases the global search ability and avoids the premature convergence; by making use of the knowledge guidance of the cultural algorithm, it improves the search efficiency and precision.

\section{The Principle of the Cultural Algorithm}

The cultural algorithm framework consists of the population space and the belief space which simulate the evolutionary process from the microscopic and macroscopic levels separately as shown in Figure 1. The former is the solution space 


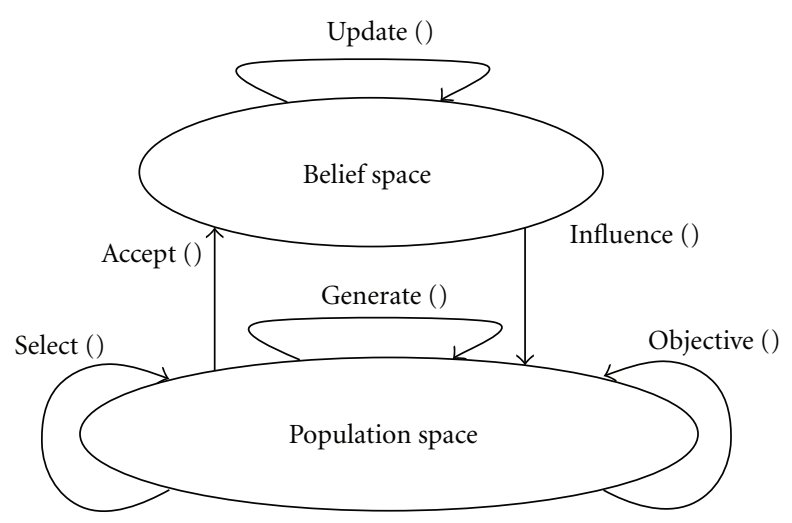

Figure 1: The framework of cultural algorithm.

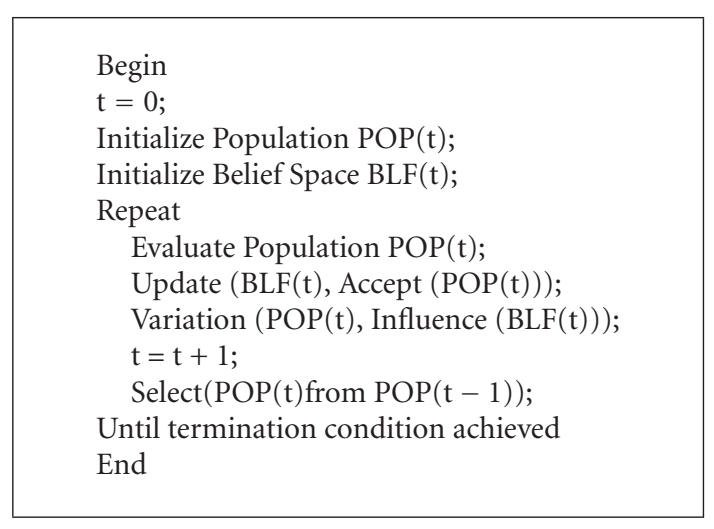

Algorithm 1 of questions, while the latter is used for forming, storing, and delivering knowledge experiences. They are mutually independent and interrelated and exchange information with each other through specific protocol. First of all, the function of accept () delivers the excellent individual experience in the evolution of the population space to the belief space, then the function of update () renews the population experience or knowledge based on the existing and new experiences in the belief space; subsequently, the function of influence () guides the evolution of the population space by means of population experience or knowledge. Furthermore, the objective function of objective () is utilized to evaluate the individual fitness values in the population space. The function of generate () forms the next-generation individuals in light of the population experience. And based on certain rules, the function of select () selects part of the newly generated individuals as their own parent. The pseudocode of the cultural algorithm is illustrated in Algorithm 1 [5].

\section{The Design of the Chaos Cultural Algorithm}

In this study, the chaotic search optimization [6] is embedded into the cultural algorithm model, and then, the chaos cultural algorithm comes into being by using the interval schemata proposed by Chung [7] and Chung and Reynolds [8] to express the knowledge in the belief space. In this way, the global search ability of the chaotic search and the experience knowledge from the iterative process are used to direct the efficient search of the algorithm to improve the search efficiency. In addition, this algorithm adopts the parallel structure of the chaotic search to efficiently reduce its sensitivity to the initial values. Without loss of generality, the issue of the nonlinear functions optimization can be described as follows:

$$
\begin{aligned}
& \min f\left(x_{i}\right), \\
& \text { s.t. } a_{i} \leq x_{i} \leq b_{i}, \quad i=1, \ldots, n
\end{aligned}
$$

3.1. Chaotic Search Optimization. As a universal nonlinear phenomenon, chaos is of stochastic, ergodic, and regular properties, where ergodicity means that the chaotic serial traverses all the states nonrepeatedly in a certain range.
In optimization, ergodicity can be used as a kind of optimization mechanism to effectively avoid the local optimum, just on which the chaotic search optimization is proposed. Bing and Weisun [6] raised a chaotic search algorithm by use of the logistic chaos mapping (see (2)) and the second carrier wave, which attracts widespread attention and causes numerous researches. This algorithm is strong in global search, but it is sensitive to the initial values and poor in the search efficiency. Some papers are aimed to improve the chaotic search optimization method, just like P-Chaos presented by Liang and $\mathrm{Gu}$ [9]. It starts parallel computing from groups of initial points and conducts double frequency carrier after searching to some extent to find the optimal solution as soon as possible. But P-chaos can hardly ensure a point is in the neighborhood of real optimal solution after first carrier, which is the foundation of the second carrier. Also, the initial searching range is relatively large, while the precise search is required later on. Last but not least, the searching is always along one side of previous one, equally one-side search failing in global search. As a result, there is still more work in improving the chaotic search optimization

$$
z^{k+1}=u * z^{k}\left(1-z^{k}\right), \quad k=0,1,2, \ldots, 0 \leq z^{k} \leq 1, u=4
$$

In the above formula, when $u=4$, the logistic mapping is chaotic and the interval $(0,1)$ is the chaos invariant set $[10]$.

The underlying idea of the chaos search optimization is to transform the chaotic state into the optimization variables by means of similar carrier, enlarge the traversing scope of the chaotic motion to the value range of the optimization variable, and then search by using the chaotic variable [6]. The basic steps are as follows.

Step 1. Initialization. Set $k=1, i=1,2, \ldots, n$, the maximum times of chaotic motion as M. Assign $n$ different initial values $z_{i}^{0}$ to the chaotic variable $z$ in (2) (the value cannot be the fixed points of the logistic mapping: $0.25,0.5,0.75)$. The chaotic variable $z_{i}^{0}$ is mapped to the value range of the optimization variables according to (3) and the initial 
optimization variable $x_{i}^{0}$ is obtained. Set $x^{*}=x^{0}, f^{*}=$ $f\left(x^{0}\right)$.

$$
x_{i}=a_{i}+z_{i} *\left|b_{i}-a_{i}\right| .
$$

Step 2. Calculate the chaotic variable sequence $z_{i}^{k}$ and the optimization variable sequence $x_{i}^{k}$.

Step 3. If $k \leq M$, then judge $f\left(x^{k}\right) \leq f^{*}$ : if the inequality holds, set $x^{*}=x^{k}, f^{*}=f\left(x^{k}\right)$; vice versa, keep $x^{*}$ and $f^{*}$ invariant and set $k=k+1$, and then turn to Step 2 . If $k>M$, end the chaotic search.

After a certain number of iteration, the logistic mapping $(u=4)$ distributes a large number of track points (chaotic sequence) in the interval $(0,1)$ and its probability density function $\rho(z)$ is [10]:

$$
\rho(z)=\frac{1}{\pi \sqrt{z(1-z)}} .
$$

This formula is the Chebyshev distribution as shown in Figure 2.

It can be seen from Figure 2 that the distribution features of logistic mapping chaotic sequence is that it is even at the middle, plenty at both sides, and up to the infinite many at the two ends. By applying this distribution feature and combining with the cultural algorithm superior interval (discussed later), each decision variable of the individuals in the population space can distribute near to the two ends of the superior interval so as to reduce the blindness search and improve the search efficiency.

3.2. The Structure of Belief Space. The structural definition of belief space is very important in the cultural algorithm framework. The primary function is to provide a definite mechanism for acquiring, storing, and delivering knowledge experience. The two kinds of most fundamental knowledge of the cultural algorithm is applied into this paper: situational knowledge and normative knowledge, that is, $\mathrm{B}=$ $\langle S, N[n]\rangle$.

In the algorithm of this study, $\mathrm{S}$ is a schedule which stores the set of excellent individuals chosen from the population space at present which are the other individuals' model, that is, $S=\left\langle E_{1}, E_{2}, \ldots, E_{e}\right\rangle$. The data structure is shown in the Figure 3 [11]. Here, every excellent individual involves its own value of decision variable and the responding adaptive value, and $F\left(E_{1}\right) \geq F\left(E_{2}\right) \geq \cdots \geq F\left(E_{e}\right)$ In this paper, $e$ reads values directly from the scale popsize of the population space. When initializing, the best individual set reads from all the individuals of the initial Population Space.

There stores $n$ sets of variation intervals of decision variables, namely, superior intervals in $N[n]$. These intervals present the most possible range of the optimal solution, which leads the chaotic iteration to arrive at the optimal

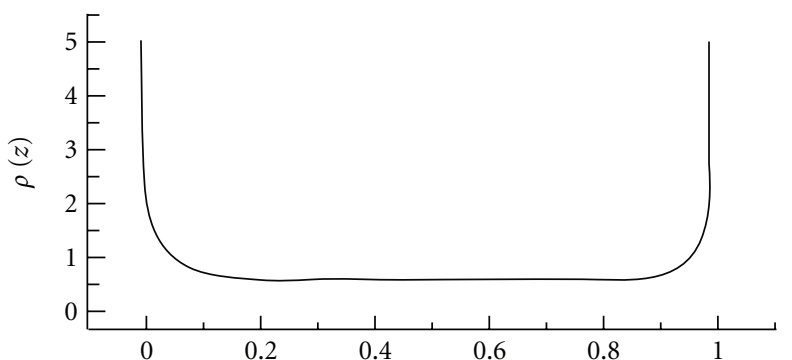

Figure 2: Probability density function of chaotic sequence of logistic map.

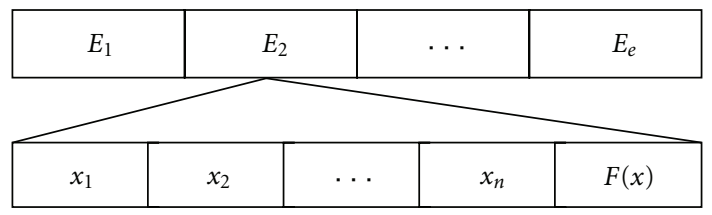

Figure 3: Data structure of situational knowledge.

solution as soon as possible. The data structure is described in Figure 4.

$$
\begin{gathered}
N[j]=\left\langle I_{j}, L_{j}, U_{j}\right\rangle, \\
I_{j}=\left[l_{j}, u_{j}\right]=\left\{x \mid l_{j} \leq x \leq u_{j}, x \in R\right\}, \quad j=1,2, \ldots, n .
\end{gathered}
$$

Therein, $l_{j}$ and $u_{j}$ represent the upper and lower boundaries of the $j$ th decision variable separately. $L_{j}$ and $U_{j}$ stand for the responding fitness values of the upper and lower boundaries, respectively. In the initial situation, $l_{j}$ and $u_{j}$ are set as the definition domain intervals, and $L_{j}$ and $U_{j}$ as $+\infty$. During the evolutionary process, these four values will adjust themselves continuously in light of the intervals of the excellent individuals.

3.3. Renovation of Belief Space. The situational knowledge in the belief space renovates twice in each generation: one happens after the chaotic search, and the other happens following the chaotic disturbance. The normative knowledge renovates once in each generation.

3.3.1. Renovation of the Situational Knowledge. The approach of renovating the situational knowledge in this paper learns from the tournament method. The specific approach is as follows.

(i) After each iteration, combine all the individual sets and the optimal individual sets represented by the situational knowledge into a new set (the scale of the new set in this algorithm is $2 *$ popsize).

(ii) Randomly select $c$ competitors in the new set (generally $c=$ popsize/2). Compare the fitness values of each individual in the new set and the $c$ competitors. Record the number of success for each 


\begin{tabular}{|l|l|l|l|}
\hline$N_{1}$ & $N_{2}$ & $\cdots$ & $N_{n}$ \\
\hline \multicolumn{3}{|c|}{} \\
\hline$l_{2}$ & $u_{2}$ & $L_{2}$ & $U_{2}$ \\
\hline
\end{tabular}

FIGURE 4: Data structure of normative knowledge.

individual. And set the number of success of the optimal fitness individual as the highest so as to make sure the optimal individual stored in the situational knowledge.

(iii) According to the number, select the top $e$ individuals (here, $e=$ popsize) as the new situational knowledge.

3.3.2. Renovation of Normative Knowledge. Suppose the $i$ th individual effect the lower boundary of the $j$ th decision variable and the $k$ th individual effect the upper boundary of the $j$ th decision variable, and then, the upper and lower boundaries of $j$ and the responding fitness values are separately showed as follows:

$$
\begin{aligned}
l_{j}^{k+1} & = \begin{cases}x_{i, j}^{k} & \text { if } x_{i, j}^{k} \leq l_{j}^{k} \text { or } F\left(x_{i}^{k}\right)<L_{j}^{k}, \\
l_{j}^{k} & \text { otherwise, }\end{cases} \\
L_{j}^{k+1} & = \begin{cases}f\left(x_{i}^{k}\right) & \text { if } x_{i, j}^{k} \leq l_{j}^{k} \text { or } F\left(x_{i}^{k}\right)<L_{j}^{k}, \\
L_{j}^{k} & \text { otherwise, }\end{cases} \\
u_{j}^{k+1} & = \begin{cases}x_{i, j}^{k} & \text { if } x_{i, j}^{k} \geq u_{j}^{k} \text { or } F\left(x_{i}^{k}\right)<U_{j}^{k}, \\
u_{j}^{k} & \text { otherwise, }\end{cases} \\
U_{j}^{k+1} & = \begin{cases}f\left(x_{i}^{k}\right) & \text { if } x_{i, j}^{k} \geq u_{j}^{k} \text { or } F\left(x_{i}^{k}\right)<U_{j}^{k}, \\
U_{j}^{k} & \text { otherwise. }\end{cases}
\end{aligned}
$$

3.4. Accept Function. The population space delivers the individual experience to the belief space by the accept function. In this way, it actually provides a group of optimal subsets to the belief space. In the case of optimization, it is usual to select the top several individuals in a linear order by a certain percentage (generally $20 \%-25 \%$ ). Also, set the dynamic accept function [12] and fuzzy accept function $[13,14]$ according to different questions. Because of the application of tournament method to the renovation of the situational knowledge in this study, the processing of the accept function has also been changed to some extent. In the renovation of the situational knowledge, the accept function accepts all the individuals at present; in the renovation of the normative knowledge, the accept function acquires the TOP several of the situational knowledge (here, TOP $=$ popsize $*$ $25 \%)$.

3.5. Influence Function. In the iterative process, the knowledge in the belief space influences the optimization variables in two ways: (1) The chaotic search at the superior interval represented by the normative knowledge; (2) The chaotic disturbance at the excellent individuals represented by the situational knowledge.

3.5.1. Chaotic Search by Using the Normative Knowledge. The normative knowledge stores the most possible range of the optimal solution which is described with the superior interval $\left(l_{j}, u_{j}\right)$. The chaotic variable caused by the logistic map is owned with the characteristics of "many at the two sides, few at the middle" at the interval $(0,1)$. In this paper, based on the responding superior interval $\left(l_{j}, u_{j}\right)$, every decision variable $j$ of the individuals is the value range of $\left(a_{j}, b_{j}\right)$ is classified into three intervals: $\left(a_{j}, l_{j}\right)$, $\left(l_{j}, u_{j}\right),\left(u_{j}, b_{j}\right)$. And then, the chaotic variables are randomly mapped to one of the three intervals. By doing this, any decision variable of the new individuals will be mainly distributed near the superior interval so as to direct the chaotic search by using the normative knowledge

$$
x_{i, j}= \begin{cases}a_{j}+z_{i} *\left|a_{j}-l_{j}\right| & \text { if random }()=0, \\ l_{j}+z_{i} *\left|l_{j}-u_{j}\right| & \text { if } \operatorname{random}()=1, \\ u_{j}+z_{i} *\left|u_{j}-b_{j}\right| & \text { if } \operatorname{random}()=2 .\end{cases}
$$

3.5.2. Chaotic Disturbance by Using the Situational Knowledge. The situational knowledge is a list storing the excellent individuals at present that is the model of the other individuals so as to be able to direct the generation of the other individuals. The length of the list is just the scale of the population space. In the algorithm of this study, the situational knowledge is the excellent individual experience formed from the chaotic iteration and stores the specific position of the excellent individuals. Therefore, it makes up for the loss of global superiority because of the restriction of the normative knowledge to the chaotic variables, so the situational knowledge possesses strong global guidance ability and plays the escaping role in the searching process.

This study firstly carries on the chaotic search, secondly renovates the situational knowledge and obtains some excellent individuals, then conducts the chaotic disturbance near the excellent individuals which is helpful for picking out better individuals and acquires the global distribution information of the optimal solution at present. As a result, it is effective to avoid being trapped in the local optimal solution. The specific description is as follows:

$$
x_{i, j}=e_{i}+\left(z_{i}-0.5\right) *\left|u_{j}-l_{j}\right| .
$$

3.6. Process of the Chaos Cultural Algorithm. In summary, this section describes the process of the proposed chaos cultural algorithm in this study to solve the optimization problem of the nonlinear function as follows.

Step 1. $\mathrm{t}=0$

Step 2. Initialize the chaotic variable and generate randomly the initial chaotic variable which cannot be the fixed point of the logistic mapping: $0.25,0.5$ and 0.75 . 
TABLE 1: Comparison of CAA and others.

\begin{tabular}{|c|c|c|c|c|c|c|c|c|c|c|}
\hline \multirow{2}{*}{ Function } & \multirow{2}{*}{$f^{*}$} & \multicolumn{3}{|c|}{ Algorithm 1} & \multicolumn{3}{|c|}{ Algorithm 2} & \multicolumn{3}{|c|}{ This algorithm } \\
\hline & & $\overline{f^{*}}$ & $\bar{t}$ & $v$ & $\overline{f^{*}}$ & $\bar{t}$ & $v$ & $\overline{f^{*}}$ & $\bar{t}$ & $v$ \\
\hline$F_{1}$ & -1.002 & -0.154877 & 2865 & 40 & -0.526565 & 1626 & 53 & -1.002 & 451 & 100 \\
\hline$F_{2}$ & -1 & -0.998745 & 965 & 80 & -0.996437 & 445 & 76 & -1 & 80 & 100 \\
\hline$F_{3}$ & 0 & 0.148157 & 1824 & 73 & 0.019447 & 528 & 90 & 0 & 596 & 93 \\
\hline$F_{4}$ & 0 & 0.284856 & 6478 & 0 & 0 & 5733 & 100 & 0 & 6547 & 100 \\
\hline$F_{5}$ & 0 & 68962.5 & 5156 & 0 & 0.014398 & 10675 & 56 & 0 & 6189 & 100 \\
\hline$F_{6}$ & 0.002288 & 0.384578 & 25157 & 0 & 0.006874 & 25345 & 0 & 0.002846 & 25247 & 10 \\
\hline
\end{tabular}

Step 3. Initialize the population space and the relevant parameters.

Step 4. Initialize the situational knowledge and normative knowledge in Belief Space.

Step 5. Evaluate the individual fitness in the population space: if meeting the requirement, terminate the process; if not, continue the process.

Step 6. Renovate the situational knowledge and normative knowledge in the belief space.

Step 7. Iterative search of the chaotic variables. Generate the new chaotic variables according to (2).

Step 8. Under the lead of the normative knowledge in the belief space, conduct the chaotic search according to (7).

Step 9. Evaluate the fitness of individuals in the population space and renovates the situational knowledge in the belief space.

Step 10. Under the guidance of the situational knowledge in the Belief Space, conduct the chaotic disturbance according to $(8)$.

Step 11. $\mathrm{t}=\mathrm{t}+1$.

Step 12. Turn to Step 5 until meeting the terminating conditions.

\section{Case Study}

The chaos cultural algorithm combines the advantages of both the chaotic search optimization and the cultural algorithm. For convenient comparison, this paper selects 6 functions from $[15,16]$ to make the optimization design and make comparisons with the parallel chaotic optimization algorithm (Algorithm 1) based on the information exchange of competition-cooperation in [16] and with the cultural algorithm (Algorithm 2) based on the evolution planning in [15]. Both Algorithms 1 and 2 are forefront in their respective fields and able to solve the optimization problem of functions effectively. The algorithm in this study takes the scale of the population space as popsize $=20$. Each algorithm runs 30 times independently. The optimization function is as follows [15-18]:

$$
\begin{aligned}
& F_{1}=-0.002-\sum_{j=1}^{25} \frac{1}{j+\sum_{i=1}^{2}\left(x_{i}-a_{i j}\right)^{6}}, \\
& -65536 \leq x_{i} \leq 65536 \text {, } \\
& {\left[a_{i j}\right]=\left[\begin{array}{ccccccccc}
-32 & -16 & 0 & 16 & 32 & -32 & \cdots & 16 & 32 \\
-32 & -32 & -32 & -32 & -32 & -16 & \cdots & 32 & 32
\end{array}\right] \text {, }} \\
& F_{2}=\frac{\sin ^{2} \sqrt{x_{1}^{2}+x_{2}^{2}}-0.5}{\left(1+0.001\left(x_{1}^{2}+x_{2}^{2}\right)\right)^{2}}-0.5, \quad-100 \leq x_{i} \leq 100, \\
& F_{3}=\left(x_{1}^{2}+x_{2}^{2}\right)^{0.25}\left[\sin ^{2} 50\left(\left(x_{1}^{2}+x_{2}^{2}\right)^{0.1}\right)+1\right] \text {, } \\
& -100 \leq x_{i} \leq 100 \text {, } \\
& F_{4}=-20 \exp \left(-0.2 \sqrt{\frac{1}{n} * \sum_{i=1}^{n} x_{i}^{2}}\right) \\
& -\exp \left(\frac{1}{n} * \sum_{i=1}^{n} \cos 2 \pi x_{i}\right)+20+e \\
& -32.768 \leq x_{i} \leq 32.768 \text {, } \\
& F_{5}=\frac{1}{4000} \sum_{i=1}^{n} x_{i}^{4}-\prod_{i=1}^{n} \cos \left(\frac{x_{i}}{\sqrt{i}}\right)+1, \quad-600 \leq x_{i} \leq 600 \text {, } \\
& F_{6}=\sum_{i=1}^{30}\left(\sum_{j=1}^{5}\left(j a_{i}^{j-1} x_{j+1}\right)-\left[\sum_{j=1}^{6}\left(a_{i}^{j-1} x_{j}\right)\right]^{2}-1\right)^{2}+x_{1}^{2} \\
& a_{i}=\frac{i-1}{29}, \quad-2 \leq x_{i} \leq 2 \text {. }
\end{aligned}
$$

Here, $F_{1}$ is the large-variable interval function; $F_{2}$ and $F_{3}$ are the functions with local extreme points; $F_{4}$ and $F_{5}$ are high-dimension complex functions with the dimension up to $30 . F_{6}$ is a complex function whose optimization point is difficult to obtain by the general methods. The comparative results between the chaos cultural algorithm and the other algorithms are shown in the Table 1 ( $f^{*}$ is the real optimal value; $\overline{f^{*}}$ stands for the average optimal value of the 30 operations; $\bar{t}$ is the average time of the 30 operations with 
millisecond as the unit; $v$ is the superiority ratio, that is, the frequency percentage of obtaining the optimal values).

It is shown in Table 1 that the algorithm adopted in this paper is much better than the other two algorithms. Algorithm 1 takes long time and has difficulty in dealing with the high-dimension and large-variable intervals. Algorithm 2 is easy to be trapped in the local extreme points so that the stability is not high. The chaos cultural algorithm not only overcomes such deficiencies, but also is superior to the other algorithms in search efficiency and precision.

\section{Conclusions}

In this paper, the authors introduce the chaotic behavior in chaotic search optimization to cultural algorithm and presents a new type of Intelligent optimization algorithms chaos cultural algorithm, the marriage of chaotic search and cultural algorithm. The chaos cultural algorithm makes use of the ergodic and stochastic properties of the chaotic search optimization so as to increase the global search ability and makes use of knowledge guidance of the cultural algorithm to decrease the frequency of the blindness search to improve the search efficiency and precision and to increase the generality. Based on the parallel structure, this algorithm adopts the chaotic search led by the normative knowledge and the chaotic disturbance led by the situational knowledge, both of which perform interactively. Therefore, this algorithm is effective to reduce the sensitivity to the initial values and enhance the stability and improve the overall performance.

In solving chaos-cultural-algorithm-based unconstrained optimization problem, the authors come up with phenomenological approach, solution procedures and algorithm flow, which is tested and verified from and experimental viewpoint. The result shows that the chaos cultural algorithm takes the advantages of both chaotic search and cultural algorithm in solving optimization problem to achieve mutual complementation. The algorithm boasts not only higher solving efficiency and precision overall, but also successfully avoiding search converge to local optimal solution prematurely.

\section{Acknowledgments}

This work is supported by National Natural Science Foundation of China (Grant no. 71171135), by the Key Discipline of Shanghai (III) (Grant no. S30504), and by the Innovation Fund Project For Graduate Student of Shanghai (Grant no. JWCXSL1001).

\section{References}

[1] R. G. Reynolds, "An introduction to cultural algorithms," in Proceedings of the 3rd Annual Conference on Evolutionary Programming, pp. 131-139, World Scientific Publishing, San Diego, Calif, USA, 1994.

[2] C. A. Coello and R. I. Becerra, "Evolutionary mutilobjective optimization using a cultural algorithm," in Proceedings of the IEEE Swarm Intelligence Symposium, pp. 6-13, IEEE Press, Indianapolis, Ind, USA, 2003.
[3] S. Saleem and R. Reynolds, "Cultural Algorithms in dynamic environments," in Proceedings of the 2000 Congress on Evolutionary Computation, pp. 1513-1520, July 2000.

[4] X. H. Yuan and Y. B. Yuan, "Application of cultural algorithm to generation scheduling of hydrothermal systems," Energy Conversion and Management, vol. 47, no. 15-16, pp. 21922201, 2006.

[5] R. G. Reynolds and S. Zhu, "Knowledge-based function optimization using fuzzy cultural algorithms with evolutionary programming," IEEE Transactions on Systems, Man, and Cybernetics, Part B: Cybernetics, vol. 31, no. 1, pp. 1-18, 2001.

[6] L. Bing and J. Weisun, "Chaos optimization method and its application," Control Theory and Applications, vol. 14, no. 4, 1997 (Chinese).

[7] C. J. Chung, Knowledge-Based Approaches to Self-adaptation in Cultural Algorithms, Wayne State University, Detroit, Mech, USA, 1997.

[8] C. J. Chung and R. G. Reynolds, "A testbed for solving optimization problems using cultural algorithms," in Proceeding of the 4th Annual Conference on Evolutionary Programming, pp. 225-236, MIT Press, Cambridge,Mass, USA, 1996.

[9] H. Y. Liang and X. S. Gu, "A novel chaos optimization algorithm based on parallel computing," Journal of East China University of Science and Technology, vol. 30, no. 4, pp. 450453, 2004.

[10] D. X. Yang, G. Li, and G. D. Cheng, "Comparative study on chaos optimization algorithm for nonlinear function," Chinese Journal of Computational Mechanics, vol. 21, no. 3, pp. 257262, 2004.

[11] C. J. Chung and R. G. Reynolds, "An evolution-based tool for real-valued function optimization using cultural algorithms," Journal on Artificial Intelligence Tools, vol. 7, no. 3, pp. 239292, 1998.

[12] S. M. Saleem, Knowledge-Based Solution to Dynamic Optimization Problems Using Cultural Algorithms, Wayne State University, Detroit, Mech, USA, 2001.

[13] S. N. Zhu and R. G. Reynolds, Fuzzy Cultural Algorithms with Evolutionary Programming VII, Springer, Berlin, Germany, 1998.

[14] S. N. ZHU, Fuzzy Cultural Algorithms with Evolutionary Programming for Real-Valued Function Optimization, Wayne State University, Detroit, Mech, USA, 1998.

[15] X. F. Yuan, Y. N. Wang, and L. H. Wu, "Parallel chaotic optimization algorithm based on competitive-cooperative inter-communication," Control and Decision, vol. 22, no. 9, pp. 1027-1031, 2007 (Chinese).

[16] C. J. Chung and R. G. Reynolds, "Knowledge-based selfadaptation in evolutionary search," International Journal of Pattern Recognition and Artificial Intelligence, vol. 14, no. 1, pp. 19-33, 2000.

[17] C. A. Coello and R. L. Becerra, "Efficient evolutionary optimization through the use of a cultural algorithm," Engineering Optimization, vol. 36, no. 2, pp. 219-236, 2004.

[18] A. D. Belegundu, Study of Mathematical Programming Methods for Structural Optimization, University of Iowa, Iowa, USA, 1982. 

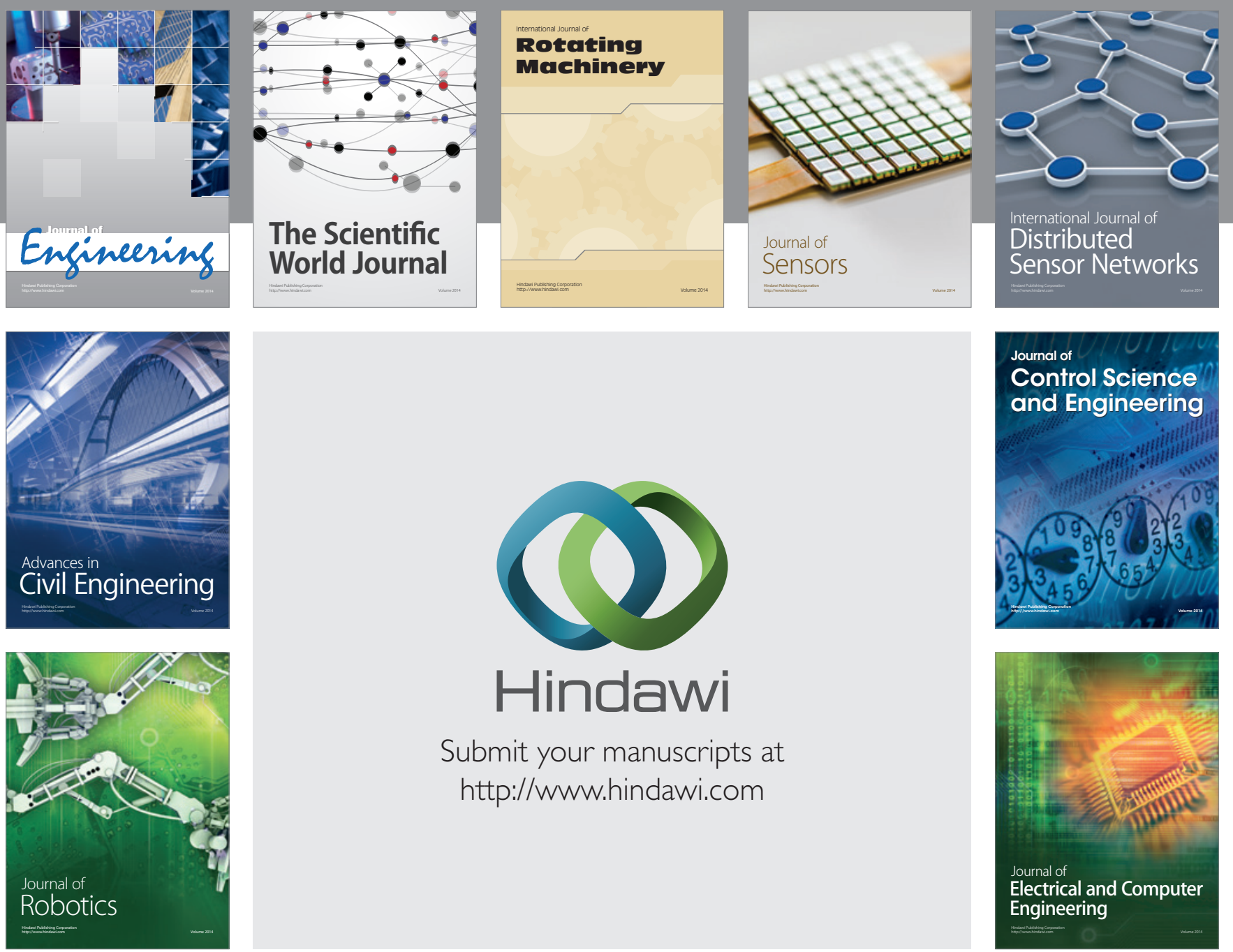

Submit your manuscripts at

http://www.hindawi.com
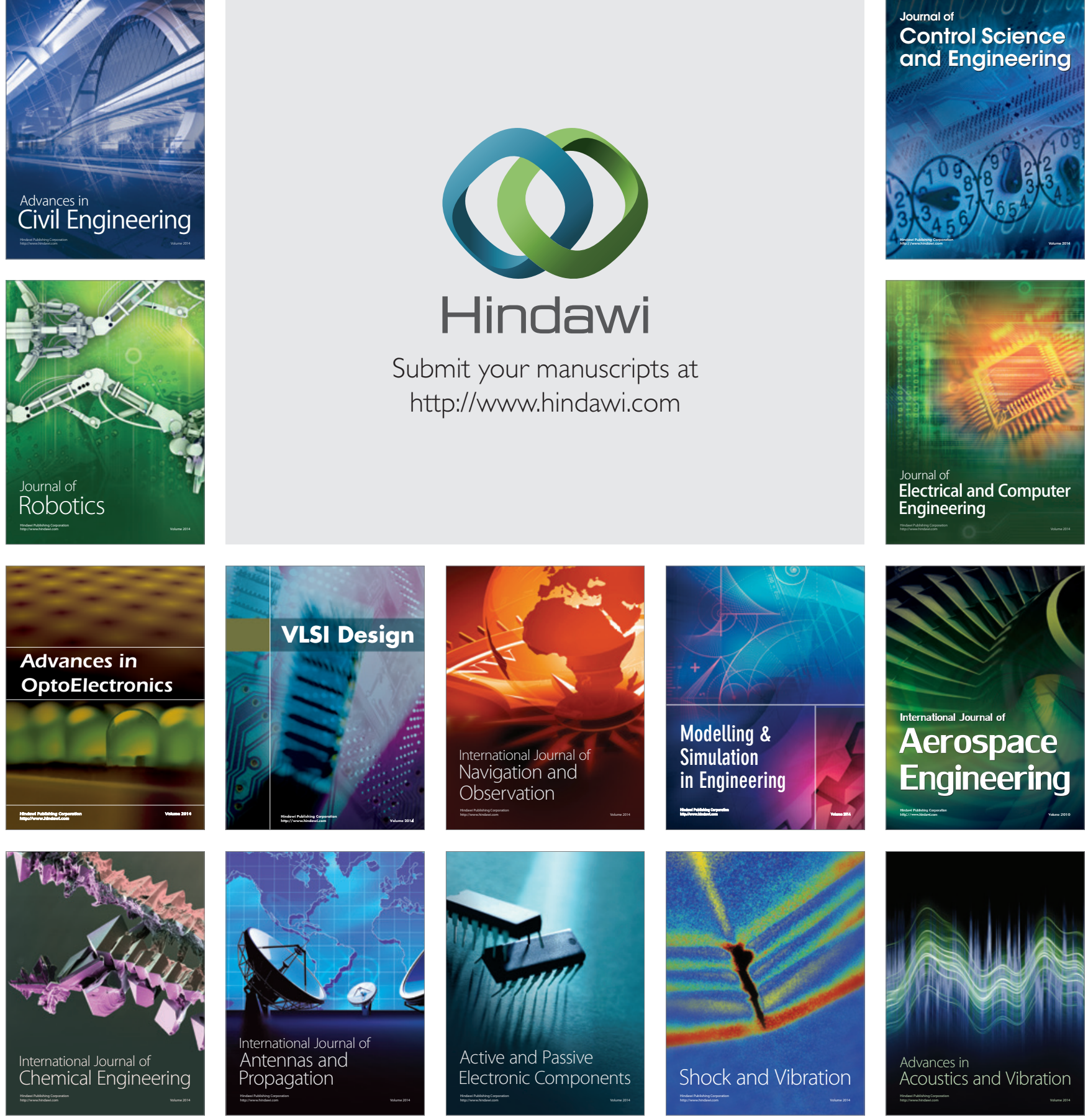